\title{
Multi-Attribute Decision-Making in Individual and Social Choice*
}

\author{
WALTER Bossert \\ Department of Economics, University of Nottingham \\ University Park, Nottingham NG7 2RD, UK \\ E-mail: lezwb@unix.ccc.nottingham.ac.uk \\ HANS PETERS \\ Department of Quantitative Economics, University of Maastricht \\ P.O. Box 616, 6200 MD Maastricht, The Netherlands \\ E-mail: h.peters@ke.unimaas.nl
}

April 1998

\begin{abstract}
This paper provides an analysis of individual and social decision criteria for alternatives that are composed of several attributes. We derive additive and multiplicative criteria for individual decision-making with new axioms and apply these criteria to obtain new justifications of known social choice rules with a bargaining interpretation, namely, the generalized utilitarian and Nash social choice functions. Unlike most axiomatizations of bargaining solutions, our approach is, to a large extent, based on the multi-attribute structure of the underlying alternatives and the resulting individual decision criteria instead of axioms that impose restrictions on the choice function directly. Journal of Economic Literature Classification Nos.: D81, D71, C78.
\end{abstract}

* We thank the Nederlandse Organisatie voor Wetenschappelijk Onderzoek (NWO) for financial support under grant no. B46-363. 


\section{Introduction}

A standard cooperative bargaining solution as introduced by Nash (1950) assigns a utility vector to each pair of a utility possibilities set and a disagreement point (the utility vector resulting if the agents fail to reach an agreement) within a given domain. For example, a typical domain of a bargaining solution consists of all pairs such that the disagreement point is normalized to be the origin and the utility possibilities set is convex and compact. Comprehensiveness of the feasible set frequently is required as well. In this paper, we consider an informationally richer framework by allowing for the possibility that the structure of the set of objects over which the bargaining process takes place may matter. In particular, we examine multi-attribute bargaining problems. A typical example for a multi-attribute bargaining problem is a wage-employment bargaining model such as the one developed by McDonald and Solow (1981).

To begin with, we provide new axiomatic derivations of additive and multiplicative multi-attribute criteria for an individual decision maker. The preferences of a decision maker over lotteries of attribute combinations are additive if they can be represented by a von-Neumann-Morgenstern (vNM) utility function ${ }^{1}$ that can be written as the sum of the utilities of the individual attributes. The multiplicative decision criterion is based on the product of the utilities over attributes. Clearly, these types of preferences possess separability properties with respect to the attributes under consideration.

Specifically, we use a weak version of utility independence ${ }^{2}$ together with an additivity axiom to generate an additive representation of an agent's preferences. Similarly, we show that the multiplicative decision criterion is implied by an analogous utility independence axiom and a condition regarding the existence and properties of worst possible attributes. By employing these new axioms, the paper also makes a contribution to the theory of decision-making under uncertainty in the presence of several attributes.

Turning from individual to social decision-making, we first characterize efficiency in both the additive and the multiplicative case. This efficiency criterion, together with some other assumptions, is then used to derive social decision procedures with a bargaining interpretation. In the additive case, efficiency and an independence condition regarding the restriction of choices to specific attributes lead to the generalized (not necessarily symmetric) utilitarian social choice functions, provided a mild regularity condition is satisfied. Analogously, in the multiplicative case, generalized Nash social choice functions

\footnotetext{
${ }^{1}$ See Fishburn (1970) for a comprehensive treatment of utility theory.

${ }^{2}$ See, for instance, Keeney and Raiffa (1976) and the references quoted there.
} 
are obtained. The novel aspect of those axiomatic derivations of well-known social choice functions is that much of the structure is imposed through the individual preferences rather than through axioms that operate directly on the social choice function itself. We therefore provide a decision-theoretic foundation of these commonly-used solutions.

\section{Multi-attribute preferences}

Let $\mathbb{N}$ denote the set of positive integers, and let $\mathbb{R}\left(\mathbb{R}_{+}, \mathbb{R}_{++}\right)$be the set of all (nonnegative, positive) real numbers. For $n \in \mathbb{N}, \mathbb{R}^{n}$ is the $n$-fold Cartesian product of $\mathbb{R}$. Let $M=\{1, \ldots, m\}$ be a set of $m \in \mathbb{N}$ attributes. The sets $A_{1}, \ldots, A_{m}$ are the corresponding non-empty spaces of these attributes. Let $A=\times_{j \in M} A_{j}$. For $a \in A$ and $M^{\prime} \subseteq M$, let $a_{-M^{\prime}}$ be the subvector of $a$ that contains the components in $M \backslash M^{\prime}$ only. If $M^{\prime}$ is a singleton $\{j\}$, we use the simpler notation $a_{-j}$ instead of $a_{-\{j\}}$. Analogously, for $A^{\prime} \subseteq A$ and $M^{\prime} \subseteq M, A_{-M^{\prime}}^{\prime}=\times_{j \in M \backslash M^{\prime}} A_{j}^{\prime}$, and if $M^{\prime}$ is a singleton $\{j\}$, we write $A_{-j}^{\prime}$ instead of $A_{-\{j\}}^{\prime}$.

$\mathcal{L}(A)$ is the set of all lotteries (i.e., finite probability distributions) over $A$. We identify a sure alternative $a \in A$ with a lottery that assigns probability one to $a$. Suppose there is a decision maker who has a preference relation (i.e., a complete and transitive binary relation) on $\mathcal{L}(A)$, representable by a vNM utility function $u: \mathcal{L}(A) \rightarrow \mathbb{R}{ }^{3}$ We also assume that there is a fixed $m$-tuple $a^{0} \in A$ with $u(a) \geq u\left(a^{0}\right)$ for all $a \in A$. That is, $a^{0}$ is a worst alternative in $A .^{4}$

Let $j \in M$. By fixing the $m-1$ attributes in $M \backslash\{j\}$ at given levels $a_{-j}^{\prime} \in A_{-j}$, the utility function $u$ induces a utility function $u_{j}: \mathcal{L}\left(A_{j}\right) \rightarrow \mathbb{R}$ on the set of lotteries over the attribute space $A_{j}$. It is straightforward to see that this induced utility function is again of the vNM type. For a lottery $\ell \in \mathcal{L}(A)$, the corresponding induced lottery on $A_{j}$ is denoted by $\ell_{j} \in \mathcal{L}\left(A_{j}\right)$.

The property of utility independence (see Keeney and Raiffa, 1976, in particular Chapters 5 and 6) requires that the induced utility function is independent of the specific levels of the other attributes. To define this property formally, let $\emptyset \neq A^{\prime} \subseteq A$, and let $j \in M$. $A_{j}^{\prime}$ is utility independent of $A_{-j}^{\prime}$ if there exist functions $f_{j}: A_{-j}^{\prime} \rightarrow \mathbb{R}$ and $g_{j}: A_{-j}^{\prime} \rightarrow \mathbb{R}_{++}$

\footnotetext{
${ }^{3}$ One may assume that the von-Neumann-Morgenstern (1947) or Herstein-Milnor (1953) axioms hold, so that such a $u$ exists.

${ }^{4}$ For convenience, we formulate the relevant definitions in terms of the vNM utility function $u$. It is straightforward to obtain equivalent formulations in terms of the underlying preference relation.
} 
such that, for any arbitrary $a_{-j}^{\prime} \in A_{-j}^{\prime}$,

$$
u(a)=f_{j}\left(a_{-j}\right)+g_{j}\left(a_{-j}\right) u\left(a_{j}, a_{-j}^{\prime}\right) \quad \text { for all } a \in A^{\prime} .
$$

Clearly, utility independence is a separability property. (1) means that, on the restricted domain $A^{\prime}$, lotteries over the values of attribute $j$ can be assessed without knowledge of specific values of the remaining attributes. See Keeney and Raiffa (1976) for a detailed discussion of this and related separability assumptions in this framework.

\section{Additive decision criteria}

In this section, we show that a specific utility independence requirement and a weak additivity assumption imply that $u$ must be additive.

Restricted $a^{0}$ utility independence requires that we can find a chain of $m-1$ attributes such that, for each attribute $j$ in this chain, $A_{j}$ is utility independent of all other attributes, where this independence can be restricted to attribute values of $a_{k}^{0}$ for all attributes $k$ that appear before $j$ in the chain. For simplicity of exposition, we formulate this condition for the chain consisting of the first $m-1$ attributes and leave the obvious generalization to the reader.

\section{Restricted $a^{0}$ utility independence:}

$A_{1}$ is utility independent of $A_{-1}$,

$A_{2}$ is utility independent of $\left\{a_{1}^{0}\right\} \times A_{-\{1,2\}}$,

$$
A_{m-1} \text { is utility independent of }\left\{a_{1}^{0}\right\} \times\left\{a_{2}^{0}\right\} \times \ldots \times\left\{a_{m-2}^{0}\right\} \times A_{m} \text {. }
$$

This independence axiom is considerably weaker than Keeney and Raiffa's (1976) mutual utility independence because, for each $j \in M \backslash\{1, m\}$, all attributes $k<j$ in the chain can be fixed at $a_{k}^{0}$. Moreover, only $m-1$ rather than all $m$ attributes are required to satisfy an independence condition.

The second axiom is a weakening of additive independence (see Keeney and Raiffa, 1976). Again, we state this condition for a chain involving the first $m-1$ attributes only in order to simplify notation.

\section{Restricted additive independence:}

For all $a_{-1} \in A_{-1}$, there exist $a_{1}^{\prime}, a_{1}^{\prime \prime} \in A_{1}$ such that $u\left(a_{1}^{\prime}, a_{-1}\right)+u\left(a_{1}^{\prime \prime}, a_{-1}^{0}\right)=$ $u\left(a_{1}^{\prime}, a_{-1}^{0}\right)+u\left(a_{1}^{\prime \prime}, a_{-1}\right)$ and $u\left(a_{1}^{\prime}, a_{-1}^{0}\right) \neq u\left(a_{1}^{\prime \prime}, a_{-1}^{0}\right)$ 
and

for all $a_{-\{1,2\}} \in A_{-\{1,2\}}$, there exist $a_{2}^{\prime}, a_{2}^{\prime \prime} \in A_{2}$ such that $u\left(a_{1}^{0}, a_{2}^{\prime}, a_{-\{1,2\}}\right)+$ $u\left(a_{2}^{\prime \prime}, a_{-2}^{0}\right)=u\left(a_{2}^{\prime}, a_{-2}^{0}\right)+u\left(a_{1}^{0}, a_{2}^{\prime \prime}, a_{-\{1,2\}}\right)$ and $u\left(a_{2}^{\prime}, a_{-2}^{0}\right) \neq u\left(a_{2}^{\prime \prime}, a_{-2}^{0}\right)$

and

$\vdots$

and

for all $a_{m} \in A_{m}$, there exist $a_{m-1}^{\prime}, a_{m-1}^{\prime \prime} \in A_{m-1}$ such that $u\left(a_{1}^{0}, \ldots, a_{m-2}^{0}, a_{m-1}^{\prime}, a_{m}\right)+$ $u\left(a_{m-1}^{\prime \prime}, a_{-(m-1)}^{0}\right)=u\left(a_{m-1}^{\prime}, a_{-(m-1)}^{0}\right)+u\left(a_{1}^{0}, \ldots, a_{m-2}^{0}, a_{m-1}^{\prime \prime}, a_{m}\right)$ and $u\left(a_{m-1}^{\prime}, a_{-(m-1)}^{0}\right)$ $\neq u\left(a_{m-1}^{\prime \prime}, a_{-(m-1)}^{0}\right)$.

Analogously to restricted $a^{0}$ utility independence, the requirements imposed by restricted additive independence become successively weaker as we progress along the chain of attributes. Restricted additive independence is analogous in spirit to Keeney and Raiffa's (1976) additive independence (formulated for two attributes only). Additive independence requires that there exist some attribute values $a_{1}^{\prime}, a_{1}^{\prime \prime} \in A_{1}$ and $a_{2}^{\prime}, a_{2}^{\prime \prime} \in A_{2}$ such that $u\left(a_{1}^{\prime}, a_{2}^{\prime}\right)+u\left(a_{1}^{\prime \prime}, a_{2}^{\prime \prime}\right)=u\left(a_{1}^{\prime}, a_{2}^{\prime \prime}\right)+u\left(a_{1}^{\prime \prime}, a_{2}^{\prime}\right)$ and $u\left(a_{1}^{\prime}, a_{2}^{\prime}\right) \neq u\left(a_{1}^{\prime}, a_{2}^{\prime \prime},\right)$ and $u\left(a_{1}^{\prime}, a_{2}^{\prime}\right) \neq$ $u\left(a_{1}^{\prime \prime}, a_{2}^{\prime}\right)$. Our version of the additive independence condition, in contrast, requires the existence of specific attribute values for several values of the other attributes, and the worst attribute combination $a^{0}$ must be involved. This is the case because we combine the axiom with a rather weak form of utility independence - namely, restricted $a^{0}$ utility independence-, whereas Keeney and Raiffa (1976) employ the much stronger mutual utility independence. Furthermore, note that restricted additive independence only requires two of the utility values involved to be distinct, but additive independence demands two inequalities of that kind.

The following theorem derives the additive decision criterion from the above axioms. Note that, in order to reformulate the axioms for general chains in this theorem, the same chains have to be used in both axioms. Recall that $a^{0}$ is a worst alternative, i.e., $u(a) \geq u\left(a^{0}\right)$ for all $a \in A$.

Theorem 1 Let $u$ satisfy restricted $a^{0}$ utility independence and restricted additive independence. Then $u$ can be written in the additive form

$$
u(a)=\sum_{j \in M} u\left(a_{j}, a_{-j}^{0}\right) \quad \text { for all } a \in A .
$$


Proof. Without loss of generality, assume $u$ is normalized so that $u\left(a^{0}\right)=0$. Because $A_{1}$ is utility independent of $A_{-1}$, there exist functions $f_{1}$ and $g_{1}$ such that

$$
u(a)=f_{1}\left(a_{-1}\right)+g_{1}\left(a_{-1}\right) u\left(a_{1}, a_{-1}^{0}\right) \quad \text { for all } a \in A
$$

where we chose $a_{-1}^{\prime}=a_{-1}^{0}$ in (1). Letting $a_{1}=a_{1}^{0}$ in $(2)$, we obtain $f_{1}\left(a_{-1}\right)=u\left(a_{1}^{0}, a_{-1}\right)$ for all $a \in A$ because $u\left(a^{0}\right)=0$. Therefore,

$$
u(a)=u\left(a_{1}^{0}, a_{-1}\right)+g_{1}\left(a_{-1}\right) u\left(a_{1}, a_{-1}^{0}\right) \text { for all } a \in A .
$$

Because, by restricted additive independence, there exists $a_{1} \in A_{1}$ such that $u\left(a_{1}, a_{-1}^{0}\right) \neq$ 0 , we can choose $a_{-1}=a_{-1}^{0}$ in $(3)$ and use $u\left(a^{0}\right)=0$ to obtain $g_{1}\left(a_{-1}^{0}\right)=1$.

Using restricted additive independence and (3), it follows that, for all $a_{-1} \in A_{-1}$, there exist $a_{1}^{\prime}, a_{1}^{\prime \prime} \in A_{1}$ such that

$$
g_{1}\left(a_{-1}\right)\left[u\left(a_{1}^{\prime}, a_{-1}^{0}\right)-u\left(a_{1}^{\prime \prime}, a_{-1}^{0}\right)\right]=g_{1}\left(a_{-1}^{0}\right)\left[u\left(a_{1}^{\prime}, a_{-1}^{0}\right)-u\left(a_{1}^{\prime \prime}, a_{-1}^{0}\right)\right] .
$$

Because, by restricted additive independence, $u\left(a_{1}^{\prime}, a_{-1}^{0}\right) \neq u\left(a_{1}^{\prime \prime}, a_{-1}^{0}\right)$, this implies $g_{1}\left(a_{-1}\right)=$ $g_{1}\left(a_{-1}^{0}\right)=1$ for all $a \in A$. Therefore,

$$
u(a)=u\left(a_{1}^{0}, a_{-1}\right)+u\left(a_{1}, a_{-1}^{0}\right) \quad \text { for all } a \in A .
$$

Because $A_{2}$ is utility independent of $\left\{a_{1}^{0}\right\} \times A_{-\{1,2\}}$, there exist functions $f_{2}$ and $g_{2}$ such that, with $a_{2}^{\prime}=a_{2}^{0}$ in (1),

$$
u\left(a_{1}^{0}, a_{-1}\right)=f_{2}\left(a_{1}^{0}, a_{-\{1,2\}}\right)+g_{2}\left(a_{1}^{0}, a_{-\{1,2\}}\right) u\left(a_{2}, a_{-2}^{0}\right) \quad \text { for all } a \in A .
$$

Letting $a_{2}=a_{2}^{0}$ in (5) and using $u\left(a^{0}\right)=0$, we obtain $f_{2}\left(a_{1}^{0}, a_{-\{1,2\}}\right)=u\left(a_{1}^{0}, a_{2}^{0}, a_{-\{1,2\}}\right)$ for all $a \in A$. Therefore,

$$
u\left(a_{1}^{0}, a_{-1}\right)=u\left(a_{1}^{0}, a_{2}^{0}, a_{-\{1,2\}}\right)+g_{2}\left(a_{1}^{0}, a_{-\{1,2\}}\right) u\left(a_{2}, a_{-2}^{0}\right) \quad \text { for all } a \in A .
$$

By restricted additive independence, there exists $a_{2} \in A_{2}$ such that $u\left(a_{2}, a_{-2}^{0}\right) \neq 0$. Therefore, letting $a_{-\{1,2\}}=a_{-\{1,2\}}^{0}$ in (6) implies $g_{2}\left(a_{-2}^{0}\right)=1$.

By restricted additive independence and (6), for all $a_{-\{1,2\}} \in A_{-\{1,2\}}$, there exist $a_{2}^{\prime}, a_{2}^{\prime \prime} \in A_{1}$ such that

$$
g_{2}\left(a_{1}^{0}, a_{-\{1,2\}}\right)\left[u\left(a_{2}^{\prime}, a_{-2}^{0}\right)-u\left(a_{2}^{\prime \prime}, a_{-2}^{0}\right)\right]=g_{2}\left(a_{-2}^{0}\right)\left[u\left(a_{2}^{\prime}, a_{-2}^{0}\right)-u\left(a_{2}^{\prime \prime}, a_{-2}^{0}\right)\right] .
$$

By restricted additive independence, $u\left(a_{2}^{\prime}, a_{-2}^{0}\right) \neq u\left(a_{2}^{\prime \prime}, a_{-2}^{0}\right)$ and, hence, $g_{2}\left(a_{1}^{0}, a_{-\{1,2\}}\right)=$ $g_{2}\left(a_{-2}^{0}\right)=1$ for all $a \in A$. Therefore,

$$
u\left(a_{1}^{0}, a_{-1}\right)=u\left(a_{1}^{0}, a_{2}^{0}, a_{-\{1,2\}}\right)+u\left(a_{2}, a_{-2}^{0}\right) \text { for all } a \in A \text {. }
$$


Using (7) in (4), we obtain

$$
u(a)=u\left(a_{1}^{0}, a_{2}^{0}, a_{-\{1,2\}}\right)+u\left(a_{2}, a_{-2}^{0}\right)+u\left(a_{1}, a_{-1}^{0}\right) \text { for all } a \in A .
$$

Repeated application of this argument for the attributes $3, \ldots, m-1$ yields

$$
u(a)=u\left(a_{m}, a_{-m}^{0}\right)+\ldots+u\left(a_{2}, a_{-2}^{0}\right)+u\left(a_{1}, a_{-1}^{0}\right) \quad \text { for all } a \in A .
$$

An immediate consequence of Theorem 1 is that $u$ can be written as

$$
u(\ell)=\sum_{j \in M} u_{j}\left(\ell_{j}\right) \text { for all } \ell \in \mathcal{L}(A),
$$

where $u_{j}: \mathcal{L}\left(A_{j}\right) \rightarrow \mathbb{R}$ for all $j \in M$.

\section{Multiplicative decision criteria}

Keeney and Raiffa (1976) prove that if each attribute space $A_{j}$ is utility independent of $A_{-j}$, then the vNM utility function $u$ can be written as a multilinear function. ${ }^{5}$ In this section, we derive the special case of a multiplicative decision criterion with a weaker utility independence axiom and an assumption which requires the $a_{j}^{0}$ to lead to a worst alternative even when combined with some values of $a_{-j}$ other than $a_{-j}^{0}$.

To exclude degenerate cases, we assume that $u$ is not a constant function. Let $a^{*} \in A$ be such that $u\left(a^{*}\right)>u\left(a^{0}\right)$. The utility independence condition is parallel to the one in the previous section, where $a^{0}$ is replaced with $a^{*}$.

\section{Restricted $a^{*}$ utility independence:}

$A_{1}$ is utility independent of $A_{-1}$, $A_{2}$ is utility independent of $\left\{a_{1}^{*}\right\} \times A_{-\{1,2\}}$,

$$
A_{m-1} \text { is utility independent of }\left\{a_{1}^{*}\right\} \times\left\{a_{2}^{*}\right\} \times \ldots \times\left\{a_{m-2}^{*}\right\} \times A_{m} .
$$

The following axiom requires $a_{j}^{0}$ to lead to a worst alternative when combined with certain values of $a_{-j}$.

\footnotetext{
${ }^{5}$ See Keeney and Raiffa (1976, Chapters 5 and 6) for details.
} 


\section{Restricted zero independence:}

$$
\begin{gathered}
u\left(a^{\prime}\right) \geq u\left(a_{1}^{0}, a_{-1}\right) \quad \text { for all } a, a^{\prime} \in A, \\
u\left(a^{\prime}\right) \geq u\left(a_{1}^{*}, a_{2}^{0}, a_{-\{1,2\}}\right) \quad \text { for all } a, a^{\prime} \in A, \\
\vdots \\
u\left(a^{\prime}\right) \geq u\left(a_{1}^{*}, \ldots, a_{m-2}^{*}, a_{m-1}^{0}, a_{m}\right) \text { for all } a, a^{\prime} \in A .
\end{gathered}
$$

Restricted zero independence implies that $a_{1}^{0}$ is a worst-possible value for attribute one in an absolute sense: $a_{1}^{0}$ leads to a worst alternative not only combined with $a_{-1}^{0}$ but combined with any value of $a_{-1}$. The remaining restrictions imposed by the axiom are weaker because they apply only to some but not to all values of the remaining attributes.

The above two axioms lead to the multiplicative decision criterion described in the following theorem.

Theorem 2 Let $u$ satisfy restricted $a^{*}$ utility independence and restricted zero independence. Then $u$ can be written in the multiplicative form

$$
u(a)=\prod_{j \in M} u\left(a_{j}, a_{-j}^{*}\right) \quad \text { for all } a \in A
$$

Proof. Without loss of generality, assume $u$ is normalized so that $u\left(a^{0}\right)=0$ and $u\left(a^{*}\right)=1$. (9) to (11) imply that we must have

$$
\begin{gathered}
u\left(a_{1}^{0}, a_{-1}\right)=0 \text { for all } a \in A, \\
u\left(a_{1}^{*}, a_{2}^{0}, a_{-\{1,2\}}\right)=0 \quad \text { for all } a \in A, \\
\vdots \\
u\left(a_{1}^{*}, \ldots, a_{m-2}^{*}, a_{m-1}^{0}, a_{m}\right)=0 \quad \text { for all } a \in A .
\end{gathered}
$$

Because $A_{1}$ is utility independent of $A_{-1}$, choosing $a_{-1}^{\prime}=a_{-1}^{*}$ in (1) implies that there exist functions $f_{1}$ and $g_{1}$ such that

$$
u(a)=f_{1}\left(a_{-1}\right)+g_{1}\left(a_{-1}\right) u\left(a_{1}, a_{-1}^{*}\right) \text { for all } a \in A \text {. }
$$

Letting $a_{1}=a_{1}^{0}$ in (15), (12) implies $f_{1}\left(a_{-1}\right)=0$ for all $a \in A$.

Now let $a_{1}=a_{1}^{*}$ in (15). Noting that $f_{1}\left(a_{-1}\right)=0$ and $u\left(a^{*}\right)=1$, it follows that $g_{1}\left(a_{-1}\right)=u\left(a_{1}^{*}, a_{-1}\right)$ for all $a \in A$. Therefore,

$$
u(a)=u\left(a_{1}^{*}, a_{-1}\right) u\left(a_{1}, a_{-1}^{*}\right) \text { for all } a \in A
$$


Because $A_{2}$ is utility independent of $\left\{a_{1}^{*}\right\} \times A_{-\{1,2\}}$, there exist functions $f_{2}$ and $g_{2}$ such that

$$
u\left(a_{1}^{*}, a_{-1}\right)=f_{2}\left(a_{1}^{*}, a_{-\{1,2\}}\right)+g_{2}\left(a_{1}^{*}, a_{-\{1,2\}}\right) u\left(a_{2}, a_{-2}^{*}\right) \quad \text { for all } a \in A
$$

(choose $a_{-2}^{\prime}=a_{-2}^{*}$ in (1)). Letting $a_{2}=a_{2}^{0}$ in (17) and using (13), we obtain $f_{2}\left(a_{1}^{*}, a_{-\{1,2\}}\right)=$ 0 for all $a \in A$. Now let $a_{2}=a_{2}^{*}$ in (17) to obtain $g_{2}\left(a_{1}^{*}, a_{-\{1,2\}}\right)=u\left(a_{1}^{*}, a_{2}^{*}, a_{-\{1,2\}}\right)$ for all $a \in A$. Hence,

$$
u\left(a_{1}^{*}, a_{-1}\right)=u\left(a_{1}^{*}, a_{2}^{*}, a_{-\{1,2\}}\right) u\left(a_{2}, a_{-2}^{*}\right) \text { for all } a \in A .
$$

Using (18) in (16), we obtain

$$
u(a)=u\left(a_{1}^{*}, a_{2}^{*}, a_{-\{1,2\}}\right) u\left(a_{2}, a_{-2}^{*}\right) u\left(a_{1}, a_{-1}^{*}\right) \text { for all } a \in A
$$

Repeated application of this argument for the attributes $3, \ldots, m-1$ yields

$$
u(a)=u\left(a_{m}, a_{-m}^{*}\right) \ldots u\left(a_{2}, a_{-2}^{*}\right) u\left(a_{1}, a_{-1}^{*}\right) \text { for all } a \in A \text {. }
$$

Again, it follows that there exist vNM utility functions $u_{j}: \mathcal{L}\left(A_{j}\right) \rightarrow \mathbb{R}$ for all $j \in M$ such that

$$
u(\ell)=\prod_{j \in M} u_{j}\left(\ell_{j}\right) \quad \text { for all } \ell \in \mathcal{L}(A)
$$

\section{Efficiency}

Consider now a situation where we have $n \in \mathbb{N}$ decision makers concerned with the multiattribute alternatives in $A$. Let $N=\{1, \ldots, n\}$ denote the set of decision makers. We use $u^{i}: \mathcal{L}(A) \rightarrow \mathbb{R}$ to denote the vNM utility function of individual $i \in N$. The induced utility functions on $\mathcal{L}\left(A_{j}\right)$ are denoted by $u_{j}^{i}: \mathcal{L}\left(A_{j}\right) \rightarrow \mathbb{R}$ for all $j \in M$ and all $i \in N$. For each agent $i \in N, a^{0 i}$ and $a^{* i}$ are the alternatives corresponding to $a^{0}$ and $a^{*}$ in the single-agent case. Note that these alternatives may be agent-specific.

In this framework, it is of interest to identify those lotteries that are efficient in the sense that it is impossible to make everyone in $N$ better off. Formally, a lottery $\hat{\ell} \in \mathcal{L}(A)$ is efficient if and only if, for all $\ell \in \mathcal{L}(A)$, there exists $i \in N$ such that $u^{i}(\hat{\ell}) \geq u^{i}(\ell)$.

The set of efficient lotteries can be characterized in the additive and multiplicative cases discussed in the previous sections. If all agents' preferences have an additive representation, we obtain the following result. Let $\Delta^{n}$ denote the unit simplex in $\mathbb{R}$. 
Theorem 3 Let each $u^{i}$ satisfy restricted $a^{0 i}$ utility independence and restricted additive independence. Then a lottery $\hat{\ell} \in \mathcal{L}(A)$ is efficient if and only if there exists $t=\left(t^{1}, \ldots, t^{n}\right) \in \Delta^{n}$ such that $\hat{\ell}$ maximizes $\sum_{i \in N} t^{i} u^{i}(\ell)$ on $\mathcal{L}(A)$ and, for all $j \in M, \hat{\ell}_{j}$ maximizes $\sum_{i \in N} t^{i} u_{j}^{i}\left(\ell_{j}\right)$ on $\mathcal{L}\left(A_{j}\right)$.

Proof. Because the set of utility vectors generated by the lotteries in $\mathcal{L}(A)$ is convex, $\hat{\ell} \in \mathcal{L}(A)$ is efficient if and only if there exists $t \in \Delta^{n}$ such that $\hat{\ell}$ maximizes $\sum_{i \in N} t^{i} u^{i}(\ell)$ on $\mathcal{L}(A)$.

Suppose $\hat{\ell} \in \mathcal{L}(A)$ maximizes $\sum_{i \in N} t^{i} u^{i}(\ell)$ on $\mathcal{L}(A)$ for some $t \in \Delta^{n}$. By $(8)$,

$$
u^{i}(\ell)=\sum_{j \in M} u_{j}^{i}\left(\ell_{j}\right) \quad \text { for all } i \in N, \text { for all } \ell \in \mathcal{L}(A)
$$

Because

$$
\max \left\{\sum_{i \in N} t^{i} \sum_{j \in M} u_{j}^{i}\left(\ell_{j}\right)\right\}=\sum_{j \in M} \max \left\{\sum_{i \in N} t^{i} u_{j}^{i}\left(\ell_{j}\right)\right\}
$$

$\hat{\ell}_{j}$ maximizes $\sum_{i \in N} t^{i} u_{j}^{i}\left(\ell_{j}\right)$ for all $j \in M$.

Conversely, suppose $\hat{\ell}_{j}$ maximizes $\sum_{i \in N} t^{i} u_{j}^{i}\left(\ell_{j}\right)$ for all $j \in M$. Using (20), it follows that $\hat{\ell}$ maximizes $\sum_{i \in N} t^{i} u^{i}(\ell)$ on $\mathcal{L}(A)$.

In the multiplicative case, we obtain an analogous result. Analogously to Theorem 2, we assume that, for each $i \in N$, there exists $a^{* i} \in A$ such that $u^{i}\left(a^{* i}\right)>u^{i}\left(a^{0 i}\right)$.

Theorem 4 Let each $u^{i}$ satisfy restricted $a^{* i}$ utility independence and restricted zero independence. Then a lottery $\hat{\ell} \in \mathcal{L}(A)$ is efficient if and only if there exists $t=$ $\left(t^{1}, \ldots, t^{n}\right) \in \Delta^{n}$ such that $\hat{\ell}$ maximizes $\prod_{i \in N} u^{i}(\ell)^{t^{i}}$ on $\mathcal{L}(A)$ and, for all $j \in M, \hat{\ell}_{j}$ maximizes $\prod_{i \in N} u_{j}^{i}\left(\ell_{j}\right)^{t^{i}}$ on $\mathcal{L}\left(A_{j}\right)$.

Proof. Again, the convexity of the set of utility vectors generated by the lotteries in $\mathcal{L}(A)$ implies that a lottery $\hat{\ell} \in \mathcal{L}(A)$ is efficient if and only if there exists $t \in \Delta^{n}$ such that $\hat{\ell}$ maximizes $\prod_{i \in N} u^{i}(\ell)^{t^{i}}$ on $\mathcal{L}(A)$, where we use the convention $0^{0}:=1$.

Suppose $\hat{\ell}$ maximizes $\prod_{i \in N} u^{i}(\ell)^{t^{i}}$ on $\mathcal{L}(A)$ for some $t \in \Delta^{n}$. By (19),

$$
u^{i}(\ell)=\prod_{j \in M} u_{j}^{i}\left(\ell_{j}\right) \quad \text { for all } i \in N, \text { for all } \ell \in \mathcal{L}(A)
$$

and, therefore, $\hat{\ell}$ maximizes

$$
\prod_{i \in N} \prod_{j \in M} u_{j}^{i}\left(\ell_{j}\right)^{t^{i}}
$$

on $\mathcal{L}(A)$. Suppose first that there exists $k \in N$ such that $u^{k}(\hat{\ell})^{t^{k}}=0$, in which case $\prod_{i \in N} u^{i}(\ell)^{t^{i}}=0$. Note that this can occur only if $t^{k}>0$ and $u^{k}(\hat{\ell})=0$. By assumption, 
there exists $a^{* i}$ such that $u^{i}\left(a^{* i}\right)>0$ for all $i \in N$. Let $\ell^{*} \in \mathcal{L}(A)$ be such that $\ell^{*}$ assigns a positive probability to each $a^{* i}$ and zero to all other alternatives. This implies $u^{i}\left(\ell^{*}\right)>0$ for all $i \in N$ and thus $\prod_{i \in N} u^{i}\left(\ell^{*}\right)^{t^{i}}>0$, contradicting the observation that $\hat{\ell}$ maximizes $\prod_{i \in N} u^{i}(\ell)^{t^{i}}$. Therefore, this case cannot occur, and we must have $u^{i}(\hat{\ell})^{t^{i}}>0$ for all $i \in N$. Hence,

$$
\max \left\{\prod_{i \in N} \prod_{j \in M} u_{j}^{i}\left(\ell_{j}\right)^{t^{i}}\right\}=\prod_{j \in M} \max \left\{\prod_{i \in N} u_{j}^{i}\left(\ell_{j}\right)^{t^{i}}\right\}
$$

and, as in Theorem 3, it follows immediately that $\hat{\ell}_{j}$ maximizes $\prod_{i \in N} u_{j}^{i}\left(\hat{\ell}_{j}\right)^{t^{i}}$ on $\mathcal{L}\left(A_{j}\right)$ for all $j \in M$.

Now suppose $\hat{\ell}_{j}$ maximizes $\prod_{i \in N} u_{j}^{i}\left(\hat{\ell}_{j}\right)^{t^{i}}$ on $\mathcal{L}\left(A_{j}\right)$ for all $j \in M$. Because $u^{i}\left(a^{* i}\right)>0$ for all $i \in N, u_{j}^{i}\left(a_{j}^{* i}\right)>0$ for all $i \in N$ and for all $j \in M$. Letting $\ell_{j}^{*} \in \mathcal{L}\left(A_{j}\right)$ be a lottery that assigns a positive probability to each $a_{j}^{* i}$ and zero to all other alternatives, it follows that $u_{j}^{i}\left(\ell_{j}^{*}\right)>0$ for all $i \in N$ and for all $j \in M$ and, thus, $\prod_{i \in N} u_{j}^{i}\left(\ell_{j}^{*}\right)^{t^{i}}>0$ for all $j \in M$. Therefore, in order for $\hat{\ell}_{j}$ to maximize $\prod_{i \in N} u_{j}^{i}\left(\hat{\ell}_{j}\right)^{t^{i}}$, it must be the case that $u_{j}^{i}\left(\hat{\ell}_{j}\right)^{t^{i}}>0$ for all $i \in N$ and for all $j \in M$. By (21), it follows that $\hat{\ell}$ maximizes $\prod_{i \in N} u^{i}(\ell)^{t^{i}}$ on $\mathcal{L}(A)$.

\section{Multi-attribute bargaining problems}

The results of the previous sections can be illustrated by applying the additive and multiplicative criteria to specific group decision problems. For example, consider the problem of selecting outcomes from a feasible set of alternatives on the basis of the agents' preferences. Suppose the space of alternatives $A$ is given, and we want to make a selection from $A$ for all profiles of individual utility functions within a given class. ${ }^{6}$ A profile of vNM utility functions is denoted by $U=\left(u^{1}, \ldots, u^{n}\right)$. The utility possibilities set generated by the profile $U$ is given by

$$
\left\{x \in \mathbb{R}^{n} \mid \exists \ell \in \mathcal{L}(A) \text { such that } x^{i}=u^{i}(\ell) \text { for all } i \in N\right\} .
$$

Accordingly, the induced utility possibilities set for attribute $j \in M$ is

$$
\left\{x \in \mathbb{R}^{n} \mid \exists \ell_{j} \in \mathcal{L}\left(A_{j}\right) \text { such that } x^{i}=u^{i}\left(\ell_{j}\right) \text { for all } i \in N\right\} .
$$

Let $\mathcal{U}$ be a set of admissible profiles $U=\left(u^{1}, \ldots, u^{n}\right)$ of vNM utility functions. Let $2^{\mathcal{L}(A)}$ be the set of all nonempty subsets of $\mathcal{L}(A)$. A social choice function is a mapping

\footnotetext{
${ }^{6}$ Because $A$ is fixed throughout this section, we simplify notation by suppressing the dependence of the functions considered here on $A$.
} 
$c: \mathcal{U} \rightarrow 2^{\mathcal{L}(A)}$ such that, for all $U \in \mathcal{U}$ and for all $\ell, \ell^{\prime} \in c(U), u_{i}(\ell)=u_{i}\left(\ell^{\prime}\right)$ for all $i \in N$. That is, we make the assumption commonly used in cooperative models of bargaining that there is a unique utility vector associated with the selected outcomes. Interpreted as a bargaining problem, the disagreement point is given by $\left(u_{1}\left(a^{01}\right), \ldots, u_{n}\left(a^{0 n}\right)\right)=$ $(0, \ldots, 0) \cdot{ }^{7} \mathrm{~A}$ bargaining solution is a special case of a social choice function, where the only relevant features are the utility possibilities set and the disagreement point of a problem. Since this additional restriction is not necessary for the purposes of this section, we will use the more general choice function $c$ as defined above.

The social choice function $c$ induces a choice function $c_{j}: \mathcal{U} \rightarrow 2^{\mathcal{L}\left(A_{j}\right)}$ for each attribute $j \in M$. Specifically, for all $j \in M$ and all $U \in \mathcal{U}, c_{j}(U)=\left\{\ell_{j} \in \mathcal{L}\left(A_{j}\right) \mid \ell \in c(U)\right\}$. Note that we do not have to require that the same utility vector results for all chosen lotteries in the image of the induced choice functions - this property follows as a consequence of our choice independence axiom defined below.

Using the results of the previous sections, we can derive generalized utilitarian and generalized Nash social choice functions in this framework. In addition to the axioms on individual preferences leading to the additive and multiplicative representations derived above, we impose some restrictions on the choice function $c$.

First, we require $c$ to be efficient in the sense that it selects efficient lotteries only. Let $E(U)$ denote the set of efficient lotteries in $\mathcal{L}(A)$ for the profile $U \in \mathcal{U}$.

Efficiency: For all $U \in \mathcal{U}, c(U) \subseteq E(U)$.

Furthermore, we impose an independence condition regarding the choice of the individual attributes.

Choice independence: For all $j \in M$, for all $U, \hat{U} \in \mathcal{U}$, if $u_{j}^{i}=\hat{u}_{j}^{i}$ for all $i \in N$, then $u_{j}^{i}\left(\ell_{j}\right)=u_{j}^{i}\left(\hat{\ell}_{j}\right)$ for all $\ell_{j} \in c_{j}(U)$ and for all $\hat{\ell}_{j} \in c_{j}(\hat{U})$.

Choice independence requires that the selection of each attribute is independent of the values of the remaining attributes and, thus, is another separability condition. Note that choice independence implies that, for a given profile, all lotteries over a specific attribute selected by the corresponding induced social choice functions lead to the same utility vector (to see this, set $U=\hat{U}$ in the definition of choice independence).

Finally, we impose a regularity condition.

Regularity: There exist $\bar{U} \in \mathcal{U}$ and $h, k \in M$ such that $h \neq k$ and, for all $j \in\{h, k\}$, there exists a unique tangent hyperplane to the induced utility possibilities set for at-

\footnotetext{
${ }^{7}$ Recall that $a^{0 i}$ denotes the worst alternative for agent $i \in N$.
} 
tribute $j$ at the point $\left(\bar{u}_{j}^{1}\left(\bar{\ell}_{j}\right), \ldots, \bar{u}_{j}^{n}\left(\bar{\ell}_{j}\right)\right)$, where $\bar{\ell}_{j} \in c_{j}(\bar{U})$.

Regularity only requires the existence of some profile such that the tangent hyperplane to two induced utility possibilities at the utility vectors induced by the choice function is unique. Note that any bargaining solution defined on a standard domain satisfies a regularity condition of that type.

We call $c$ a generalized utilitarian social choice function if there exists $t \in \Delta^{n}$ such that, for all $U \in \mathcal{U}, c(U)$ is a subset of the set of maximizers of $\sum_{i \in N} t^{i} u^{i}(\ell)$ on $\mathcal{L}(A)$. Analogously, $c$ is a generalized Nash social choice function if there exists $t \in \Delta^{n}$ such that, for all $U \in \mathcal{U}, c(U)$ is a subset of the set of maximizers of $\prod_{i \in N} u^{i}(\ell)^{t^{i}}$ on $\mathcal{L}(A)$.

Theorem 5 Let $\mathcal{U}$ be the set of all profiles $U$ such that the utility possibilities set associated with $U$ is compact and, for each $i \in N$, there exists $a^{0 i} \in A$ such that $u^{i}(a) \geq$ $u^{i}\left(a^{0 i}\right)=0$ for all $a \in A$, and $u^{i}$ satisfies restricted $a^{0 i}$ utility independence and restricted additive independence. Let $c$ be a social choice function satisfying efficiency, choice independence, and regularity. Then $c$ is a generalized utilitarian social choice function.

Proof. Let $\bar{U} \in \mathcal{U}$ and $h, k \in M$ be as in the definition of the regularity axiom. By efficiency and the definition of $c$, there exists $t \in \Delta^{n}$ such that $\bar{\ell}$ is a maximizer of $\sum_{i \in N} t^{i} \bar{u}^{i}(\ell)$ on $\mathcal{L}(A)$ for all $\bar{\ell} \in c(\bar{U})$. By Theorem $3, \bar{\ell}_{j}$ maximizes $\sum_{i \in N} t^{i} \bar{u}_{j}^{i}\left(\ell_{j}\right)$ on $\mathcal{L}\left(A_{j}\right)$ for all $j \in M$. Regularity implies that, for $j \in\{h, k\}, t$ is the only vector of coefficients for which $\hat{\ell}_{j}$ maximizes this weighted sum on $\mathcal{L}\left(A_{j}\right)$.

Let $U \in \mathcal{U}$ be arbitrary. Let the profile $\hat{U}$ be such that $\hat{u}_{h}^{i}=\bar{u}_{h}^{i}$ and $\hat{u}_{j}^{i}=u_{j}^{i}$ for all $i \in N$ and for all $j \in M \backslash\{h\}$. Let $\hat{\ell} \in c(\hat{U})$. By efficiency, $\hat{\ell}$ maximizes $\sum_{i \in N} \hat{t}^{i} \hat{u}^{i}(\ell)$ on $\mathcal{L}(A)$ for some $\hat{t} \in \Delta^{n}$. Theorem 3 implies that $\hat{\ell}_{j}$ maximizes $\sum_{i \in N} \hat{t}^{i} \hat{u}_{j}^{i}\left(\ell_{j}\right)$ on $\mathcal{L}\left(A_{j}\right)$ for all $j \in M$. Choice independence implies that $\hat{u}_{h}^{i}\left(\hat{\ell}_{h}\right)=\hat{u}_{h}^{i}\left(\bar{\ell}_{h}\right)$. By regularity, $\hat{t}=t$.

Now let the profile $\tilde{U}$ be such that $\tilde{u}_{k}^{i}=\bar{u}_{k}^{i}$ and $\hat{u}_{j}^{i}=u_{j}^{i}$ for all $i \in N$ and for all $j \in M \backslash\{k\}$. Let $\tilde{\ell} \in c(\tilde{U})$. By efficiency, $\tilde{\ell}$ maximizes $\sum_{i \in N} \tilde{t}^{i} \tilde{u}^{i}(\ell)$ on $\mathcal{L}(A)$ for some $\tilde{t} \in \Delta^{n}$. Again, Theorem 3 implies that $\tilde{\ell}_{j}$ maximizes $\sum_{i \in N} \tilde{t}^{i} \tilde{u}_{j}^{i}\left(\ell_{j}\right)$ on $\mathcal{L}\left(A_{j}\right)$ for all $j \in M$. Choice independence implies that $\tilde{u}_{k}^{i}\left(\tilde{\ell}_{k}\right)=\tilde{u}_{k}^{i}\left(\bar{\ell}_{k}\right)$. By regularity, $\tilde{t}=t$.

Let $\ell \in c(U)$. By choice independence, for all $i \in N, u_{j}^{i}\left(\ell_{j}\right)=u_{j}^{i}\left(\hat{\ell}_{j}\right)$ for all $j \in M \backslash\{h\}$ and $u_{h}^{i}\left(\ell_{h}\right)=u_{h}^{i}\left(\tilde{\ell}_{h}\right)$. This implies that $\ell_{j}$ maximizes $\sum_{i \in N} t^{i} u_{j}^{i}\left(\ell_{j}\right)$ on $\mathcal{L}\left(A_{j}\right)$ for all $j \in M$ and, using the argument in the proof of Theorem 3 , it follows that $\ell$ maximizes $\sum_{i \in N} t^{i} u^{i}(\ell)$ on $\mathcal{L}(A)$.

Analogously, the generalized Nash social choice functions are implied in the multiplicative case. Because the proof of this result is parallel to the proof of the previous theorem, it is omitted. 
Theorem 6 Let $\mathcal{U}$ be the set of all profiles $U$ such that the utility possibilities set associated with $U$ is compact and, for each $i \in N$, there exist $a^{0 i}$, $a^{* i} \in A$ such that $u^{i}(a) \geq u^{i}\left(a^{0 i}\right)=0$ for all $a \in A$ and $1=u^{i}\left(a^{* i}\right)>u^{i}\left(a^{0 i}\right)$, and $u^{i}$ satisfies restricted $a^{* i}$ utility independence and restricted zero independence. Let $c$ be a social choice function satisfying efficiency, choice independence, and regularity. Then $c$ is a generalized Nash social choice function.

An interesting feature of Theorems 5 and 6 is that the resulting social choice functions are such that the attributes can be separated in a bargaining process: it is sufficient to consider each attribute independently of the remaining ones. This considerably facilitates the application of the procedure to actual bargaining situations.

As an illustration, suppose the allocation of $m$ goods in an $n$-agent economy is to be determined by means of a bargaining process. If the individual preferences satisfy the appropriate independence axioms, the results of this section can be used to justify the use of a generalized utilitarian solution or a generalized Nash solution. By adding a symmetry or anonymity condition, the utilitarian solution and the Nash solution are obtained.

\section{Concluding remarks}

The results of this paper provide a decision-theoretic foundation for specific social choice procedures. In particular, multi-attribute decision criteria are employed. An assumption underlying our approach is that individual preferences are of the same structure. One possibility for extending our work would be to examine situations where individual agents may have different types of utility functions over multi-attribute alternatives.

\section{References}

Fishburn, P.C. (1970): Utility Theory for Decision Making. New York: John Wiley \& Sons.

Herstein, I.N., and J. Milnor (1953): "An Axiomatic Approach to Measurable Utility," Econometrica, 21, 291-297.

Keeney, R.L., and H. Raiffa (1976): Decisions with Multiple Objectives: Preferences and Value Tradeoffs. New York: John Wiley \& Sons. 
McDonald, I.M., and R.M. Solow (1981): "Wage Bargaining and Employment," American Economic Review, 71, 896-908.

Nash, J.F. (1950): “The Bargaining Problem," Econometrica, 18, 155-162.

von Neumann, J., and O. Morgenstern (1947): Theory of Games and Economic Behavior. Princeton: Princeton University Press (2nd edition). 